\title{
A cross-sectional analysis of the relationship between uric acid and coronary atherosclerosis in patients with suspected coronary artery disease in China
}

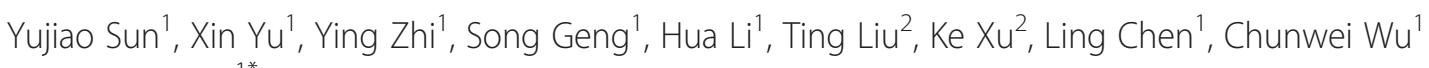
and Guoxian Qi ${ }^{1 *}$

\begin{abstract}
Background: Although many studies have examined the relationship between uric acid (UA) and coronary artery disease (CAD), whether UA is an independent risk factor contributing to progression of CAD is still controversial. Whether UA plays a different role in different sexes is also unclear.

Methods: A total of 1116 individuals with suspected CAD were stratified into four groups according to their serum UA quartiles in total (men and women combined), in men, and in women. The association of UA with coronary atherosclerosis was assessed by univariable and multivariable logistic regression.

Results: In total and in women, the prevalence of any plaques and significant/severe stenosis was significantly increased with an increase in quartiles of $U A$ (all $P<0.05$ ). The proportion of triple-vessel disease and left main artery lesion was highest in the fourth quartile (both $p<0.05$ ). Increasing quartiles of $U A$ were significantly associated with a coronary artery calcium score (CACS) $>10$ (all $P<0.01$ ). As UA levels increased in women, the incidence of double-vessel lesions $(p=0.017)$ and the proportion of mixed plaques $(p=0.022)$ were significantly increased. The proportion of a CACS of 0 in total, in men and women was highest in the first quartile (all $P<0.01$ ). UA was the strongest predictor of significant stenosis, multivessel disease, and mixed plaques in women (all $p<0.05)$. UA was the only risk factor for mixed plaques in total $(P=0.046)$.
\end{abstract}

Conclusion: The level of UA was significantly associated with coronary atherosclerosis in women, but not men.

Keywords: Uric acid, Coronary atherosclerosis, Coronary computed tomography angiography, Gender, Calcium score

\section{Background}

Uric acid (UA) is the main end product of purine catabolism [1]. High UA levels are often accompanied by obesity, hyperlipidemia, hypertension, glucose intolerance, and insulin resistance [2-5], which contribute to the development of cardiovascular disease. Elevated UA levels are associated with coronary artery disease (CAD), independently of traditional CAD risk factors [6,7].

\footnotetext{
*Correspondence: qigx2011@hotmail.com

'Department of Cardiology of Aging, Department of Cardiology, The First Affiliated Hospital of China Medical University, NO.155 Nanjing North Street, Heping Ward, Shenyang 110001, China

Full list of author information is available at the end of the article
}

However, some studies have suggested that UA is only considered as a risk marker or an adaptive ascended to attempt to prevent atherosclerosis [8-10], and this may be due to its antioxidant properties [11]. Although studies have examined the relationship between UA and CAD, whether increased UA is an independent risk factor that contributes to early CAD is still controversial.

Sex might be an important factor involved in the relationship between UA and CAD. In a subgroup analysis of LIFE [12], the relationship between UA and CAD was significant only in women. A meta-analysis [6] found that UA was significantly correlated with CAD only in women. A strong association between UA and cardiac 
events has been observed in both sexes in other studies $[13,14]$. Whether UA plays a different role in different sexes in the progression of CAD is unclear.

Therefore, in this study, we assessed the association between UA and coronary atherosclerosis in patients with suspected CAD who underwent 256-detector-row coronary computed tomographic angiography (CCTA). We further assessed these associations in sex subgroups.

\section{Methods}

\section{Study population}

This study included 5150 consecutive individuals ( $\geq 18$ years) in China undergoing CCTA and coronary artery calcium score (CACS) measurements in our institution from September 2011 to February 2013. CCTA and measurement of the CACS were performed for the suspicion of CAD after clinical assessment (including cardiac symptoms, risk factors, electrocardiogram changes, and a positive stress test). Finally, 1116 individuals were enrolled (Figure 1). All patients gave written inform consent, and the study was approved by the ethics committee of the First Affiliated Hospital of China Medical University.

\section{Assessment of CAD risk factors}

All patients were systematically asked about their demographics by professionals. Body weight, height, and blood pressure were measured. Hypertension was defined as a previously established diagnosis and/or antihypertensive medication, systolic blood pressure $\geq 140 \mathrm{mmHg}$, and diastolic blood pressure $\geq 90 \mathrm{mmHg}$. Diabetes mellitus was defined as a previously established diagnosis and/or antidiabetic treatment, and fasting glucose $\geq 126 \mathrm{mg} / \mathrm{dl}$. A family history of CAD was defined as a first-degree male relative aged $<55$ years or a first-degree female relative aged $<65$ years. Smoking was defined as any cigarette smoking within 1 year of CCTA. Medication use was recorded in detail.

Total cholesterol (TC), triglycerides (TG), high-density lipoprotein cholesterol (HDL-C), low-density lipoprotein cholesterol (LDL-C), creatinine and UA levels were measured after at least a 12-h fasting period within 7 days of CCTA. The contents of UA were measured with enzyme kinetics in all enrolled patients, and it was uniform throughout the study period.

\section{Acquisition of images}

Computed tomography scans were performed using 256-detector-row CCTA (Brilliance; Philips Medical System, The Netherlands). Individuals with a heart rate $\geq 75$ beats per minute were treated orally with up to $100 \mathrm{mg}$ metoprolol for several hours (except for contraindications of beta-blockers) before CCTA imaging to achieve a higher image quality. First, after a scout radiograph of the chest (lateral and anteroposterior), a noncontrast CACS scan was performed, and the image section thickness was $2.5 \mathrm{~mm}$ by triggering at a heart rate depending on the percentage of the $R-R$ interval. The sections were collected from the level of the carina and proceeded to the level of the diaphragm. Thereafter, retrospective electrocardiogram-gated contrast-enhanced CCTA was performed. The CCTA scan was initiated $20 \mathrm{~mm}$ above the level of the left main artery to $20 \mathrm{~mm}$ below the inferior myocardial apex during a single

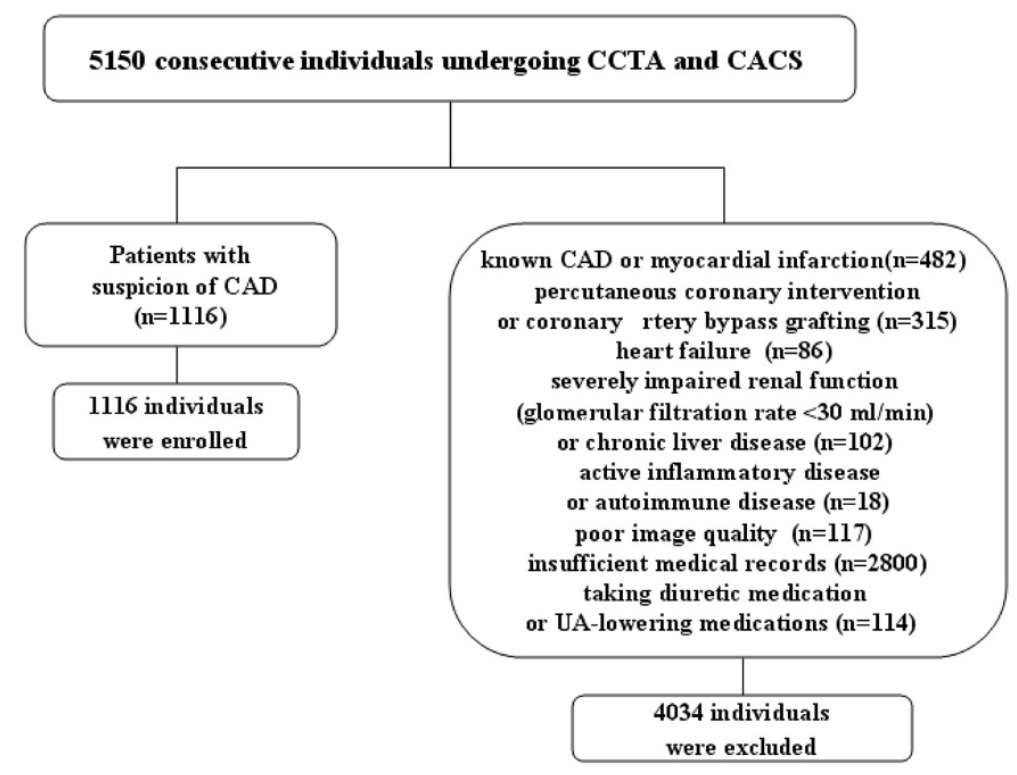

Figure 1 The path of patients enrollment. 
breath-hold. Depending on the individual's weight, a bolus of $50-80 \mathrm{ml}$ of iopamidol or iohexol was intravenously injected at $4-5.5 \mathrm{ml} / \mathrm{s}$ into the antecubital vein, followed by $50 \mathrm{ml}$ of saline. A standard scan protocol was applied, with section collimation of $256 \times 0.625 \mathrm{~mm}$, $0.27 \mathrm{~s}$ for rotation time, $120 \mathrm{KV}$ tube voltage, and 800 $1100 \mathrm{~mA}$ tube current. In all scans, electrocardiogramgate dose modulation was used. The electrocardiograms of individuals were simultaneously collected to allow for retrospective segmental data reconstruction. The images of all individuals were initially reconstructed at $75 \%$ of the R-R interval of the cardiac cycle. If motion artefacts were found, reconstruction of additional phases was performed. The best R-R interval image quality was chosen for interpretation. The dose range of radiation for CCTA was estimated to be $10-18 \mathrm{mSv}$.

\section{Image analysis}

All images were analysed separately by two experienced radiologists and one cardiologist who were blinded to the patients' characteristics. Consensus on interpretation was performed to achieve a final CCTA diagnosis. All scans were evaluated by a three-dimensional workstation (Brilliance; Philips Medical Systems). The CACS was measured using the scoring system previously described by Agatston et al. [15].

The three reads were permitted to use any/all available post-processing image reconstruction algorithms, including two-dimensional axial or three-dimensional maximal intensity projection, multiplanar reformat, cross-sectional analysis, or the volume-rendered technique. A 16-segment coronary artery tree model [16] was used in the analysis of coronary arteries. In each coronary segment, plaques were defined as any tissue structure $>1 \mathrm{~mm}^{2}$, which existed either within the coronary artery lumen or was adjacent to the coronary artery lumen, and could be discriminated from surrounding pericardial tissue, epicardial fat, or the vessel lumen itself. For evaluating the degree of stenosis, the coronary lumen was semi-automatically traced at the maximal stenosis site and was compared with the mean value of a proximal and distal reference site. The image quality was evaluated and classified as follows: good, with no artifact; adequate, with the presence of artifacts but feasible for evaluating the degree of stenosis and plaque characteristics; or poor, with the presence of artifacts and not feasible for evaluating the degree of stenosis and plaque characteristics. If an image was graded as poor, the image was not included.

Coronary lesions $\geq 50$ and $70 \%$ were defined as significant and severe, respectively. Multivessel disease was defined as the presence of stenosis of more than $50 \%$ in at least two vessels. The stenosed coronary vessel of individuals was further categorized as having one-, two-, and three-vessel/left main disease. All detected plaques were classified as calcified, non-calcified, or mixed. The calcified component of a stenosis was defined as a lesion with radiodensity greater than the luminal contrast. The non-calcified component of a stenosis was defined as a lesion with radiodensity greater than that of neighbouring soft tissue and lower than the luminal contrast. Plaques that contained calcified tissue greater than $75 \%$ of the plaque area were classified as calcified plaques, less than $25 \%$ as non-calcified plaques, and $25-75 \%$ as mixed plaques [17].

\section{Statistical analysis}

Baseline characteristics are expressed as absolute counts and proportions for categorical variables, and as means \pm standard deviations for continuous variables. Continuous variables were analysed by analysis of variance and categorical variables were analysed by $\chi^{2}$ test.

The association of UA with coronary atherosclerosis was assessed by the $\chi^{2}$ test. Univariable and multivariable logistic regression were used to assess the association of UA with significant stenosis, multivessel disease, high CACS, and plaque characteristics. Variables that showed a $\mathrm{P}$ value $<0.1$ with univariable analysis were applied to multivariate analysis. All analyses were performed using SPSS 15.0. The level of significance was set at $P<0.05$.

\section{Results}

\section{Baseline characteristic baseline characteristics}

Among the 1116 patients in the study, 50.7\% were men, and the mean age was $58.05 \pm 10.69$ years. All individuals were stratified into four groups according to their UA quartile, the first quartile: $<259 \mu \mathrm{mmol} / \mathrm{l}(\mathrm{n}=278)$; the second quartile: $259-309 \mu \mathrm{mmol} / \mathrm{l} \quad(\mathrm{n}=274)$; the third quartile: $310-373 \mu \mathrm{mmol} / \mathrm{l}(\mathrm{n}=283)$ and the fourth quartile: $>373 \mu \mathrm{mmol} / \mathrm{l}(\mathrm{n}=281)$. With increasing UA quartiles, the prevalence of male sex, smoking, and hypertension was significantly increased (all $P<0.01$ ). Body mass index (BMI) and TC level were significantly higher (all $P<0.001$ ) and HDL-C level was significantly lower $(P<0.001$, Table 1$)$. Figure 2 showed the representative view of mixed, noncalcified and calcified plaque.

\section{Prevalence, characteristics, and the CACS of coronary artery plaques}

Men and women were stratified into four groups according to their UA quartile. The quartiles of UA in men were the first quartile: $296 \mu \mathrm{mmol} / \mathrm{l}(\mathrm{n}=140)$; the second quartile: $<296-349 \mu \mathrm{mmol} / \mathrm{l}(\mathrm{n}=140)$; the third quartile: $350-406$ $\mu \mathrm{mmol} /(\mathrm{n}=144)$ and the fourth quartile: $>406 \mu \mathrm{mmol} / \mathrm{l}$ $(n=142)$. The quartiles of UA in women were the first quartile: $<238 \mu \mathrm{mmol} / \mathrm{l}(\mathrm{n}=136)$; the second quartile: 238 $273 \mu \mathrm{mmol} / \mathrm{l}(\mathrm{n}=137)$; the third quartile: $274-326 \mu \mathrm{mmol} /$ $\mathrm{l}(\mathrm{n}=138)$ and the fourth quartile $>326 \mu \mathrm{mmol} / \mathrm{l}(\mathrm{n}=139)$. With increasing UA quartiles in total (men and women 
Table 1 Baseline characteristics of the all study population according to the quartiles of the serum uric acid

\begin{tabular}{|c|c|c|c|c|c|c|}
\hline Variables & Total $(n=1116)$ & $\begin{array}{l}\text { 1st Quartile } \\
<259 \mu \mathrm{mol} / \mathrm{l} \\
(\mathrm{n}=278)\end{array}$ & $\begin{array}{l}\text { 2nd Quartile } \\
259-309 \mu \mathrm{mol} / \mathrm{l} \\
(\mathrm{n}=274)\end{array}$ & $\begin{array}{l}\text { 3rd Quartile } \\
310-373 \mu \mathrm{mol} / \mathrm{l} \\
(\mathrm{n}=283)\end{array}$ & $\begin{array}{l}\text { 4th Quartile } \\
>373 \mu \mathrm{mol} / \mathrm{l} \\
(\mathrm{n}=281)\end{array}$ & P-value \\
\hline Age(years) & $58.05 \pm 10.69$ & $58.64 \pm 9.19$ & $57.86 \pm 9.52$ & $58.14 \pm 11.09$ & $57.56 \pm 12.6$ & 0.674 \\
\hline Male & $566(50.7)$ & $64(23.0)$ & $109(39.8)$ & $170(60.1)$ & $223(79.4)$ & $<0.001$ \\
\hline $\mathrm{BMI}\left(\mathrm{kg} / \mathrm{m}^{2}\right)$ & $24.99 \pm 4.00$ & $23.46 \pm 4.24$ & $24.70 \pm 3.29$ & $25.83 \pm 4.45$ & $25.95 \pm 3.38$ & $<0.001$ \\
\hline Smoking & $279(25.0)$ & $40(14.4)$ & $59(21.5)$ & 89 (31.4) & $91(32.4)$ & $<0.001$ \\
\hline Family history of CAD & $93(8.3)$ & $27(9.7)$ & 29 (10.6) & $20(7.1)$ & $17(6.0)$ & 0.167 \\
\hline Hypertension & $639(42.7)$ & $96(34.5)$ & $114(41.6)$ & $135(47.7)$ & $132(47.0)$ & 0.005 \\
\hline Diabetes Mellitus & $183(16.4)$ & $45(16.2)$ & $43(15.7)$ & $51(18.0)$ & $44(15.7)$ & 0.861 \\
\hline $\mathrm{LDL}-\mathrm{C}(\mathrm{mmol} / \mathrm{l})$ & $2.95 \pm 0.88$ & $2.93 \pm 0.79$ & $3.02 \pm 1.01$ & $2.94 \pm 0.87$ & $2.92 \pm 0.87$ & 0.544 \\
\hline $\mathrm{HDL}-\mathrm{C}(\mathrm{mmol} / \mathrm{l})$ & $1.19 \pm 0.37$ & $1.33 \pm 0.34$ & $1.21 \pm 0.32$ & $1.13 \pm 0.30$ & $1.09 \pm 0.46$ & $<0.001$ \\
\hline $\mathrm{TC}(\mathrm{mmol} / \mathrm{l})$ & $4.60 \pm 1.06$ & $4.61 \pm 0.97$ & $4.66 \pm 1.22$ & $4.58 \pm 1.06$ & $4.56 \pm 0.99$ & 0.735 \\
\hline $\mathrm{TG}(\mathrm{mmol} / \mathrm{l})$ & $1.64 \pm 1.36$ & $1.29 \pm 0.85$ & $1.59 \pm 1.29$ & $1.69 \pm 1.47$ & $1.98 \pm 1.61$ & $<0.001$ \\
\hline Creatinine $(\mu \mathrm{mol} / \mathrm{l})$ & $78.20 \pm 16.32$ & $81.47 \pm 16.74$ & $80.09 \pm 14.23$ & $74.07 \pm 15.38$ & $81.95 \pm 19.17$ & 0.121 \\
\hline \multicolumn{7}{|l|}{ History of Medication } \\
\hline Aspirin & $247(22.1)$ & $64(23.0)$ & $57(20.8)$ & $67(23.7)$ & $59(21.0)$ & 0.799 \\
\hline Beta blcker & $118(10.6)$ & $27(9.7)$ & $26(9.5)$ & $31(11.0)$ & $34(12.1)$ & 0.733 \\
\hline ACEI & $62(5.6)$ & $13(4.7)$ & $15(5.5)$ & $17(6.0)$ & $17(5.6)$ & 0.880 \\
\hline ARB & $68(6.1)$ & $14(5.0)$ & $13(4.7)$ & $20(7.1)$ & $21(7.5)$ & 0.417 \\
\hline CCB & $145(13.0)$ & $28(10.1)$ & $43(15.7)$ & $42(14.8)$ & $32(11.4)$ & 0.144 \\
\hline Statins & $94(8.4)$ & $20(7.2)$ & $25(9.1)$ & $30(10.6)$ & $19(6.8)$ & 0.330 \\
\hline Antihyperglycemic & $107(9.6)$ & $30(10.8)$ & $23(8.4)$ & $29(10.2)$ & $25(8.9)$ & 0.750 \\
\hline Insulin & $65(5.8)$ & $18(6.5)$ & $14(5.1)$ & $18(6.4)$ & $15(5.3)$ & 0.863 \\
\hline
\end{tabular}

Values are mean \pm Standard deviation or $n(\%)$. The conversion of UA measuring unit: $\mathrm{mg} / \mathrm{dl}=(\mu \mathrm{mol} / \mathrm{l}$ divide by 59.48$)$.

$B M I$ Body Mass Index, CAD Coronary artery disease, LDL-C Low-density lipoprotein Cholesterol, HDL-C High-density lipoprotein Cholesterol, TC Total Cholesterol, TG Triglycerides, ACEI Angiotensin-converting enzyme inhibitor, ARB Angiotensin receptor blocker, CCB Calcium Channel Blocker.

combined) and in women, the prevalence of plaques were significantly increased (total: $56.8 \%$ vs $62.0 \%$ vs $70.0 \%$ vs $73.0 \%, P<0.001$; women: $47.1 \%$ vs $57.7 \%$ vs $59.4 \%$ vs $69.8 \%, \mathrm{p}=0.002$, (Figure $3 \mathrm{~A}$ ), significant stenosis were significantly increased (total: $25.5 \%$ vs $30.3 \%$ vs $39.6 \%$ vs $40.2 \%, P<0.001$; women: $16.9 \%$ vs $29.2 \%$ vs $30.4 \%$ vs $33.1 \%, P=0.010$, Figure $3 \mathrm{~B}$ ), and severe stenosis were significantly increased (total: $12.9 \%$ vs $18.2 \%$ vs $21.9 \%$ vs $25.6 \%, P=0.001$; women: $8.1 \%$ vs $16.8 \%$ vs $17.4 \%$ vs $19.4 \%$, $P=0.032$, Figure $3 C$ ).
The incidence of double-vessel lesions was only significantly increased with UA quartiles in women $(9.6 \%$ vs $13.1 \%$ vs $14.5 \%$ vs $23.0 \%, P=0.017$, Figure $3 \mathrm{E}$ ). In total and in women, the proportion of triple-vessel/left main artery lesions were highest in the fourth quartile (total: $15.8 \%$ vs $19.3 \%$ vs $26.5 \%$ vs $28.8 \%, P<0.001$; women: $13.2 \%$ vs $16.8 \%$ vs $24.6 \%$ vs $25.2 \%, P=0.027$, Figure $3 \mathrm{~F}$ ).

The proportion of a CACS of 0 in total, in men, and in women were lowest in the fourth quartile (total: $64.4 \%$ vs $59.1 \%$ vs $49.1 \%$ vs $46.6 \%, P<0.001$; men: $55.7 \%$

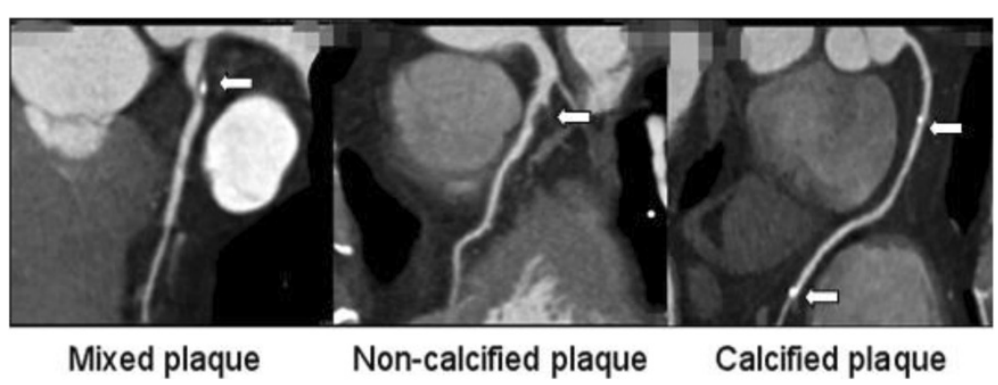

Figure 2 Showed the representative view of mixed, non-calcified plaque. 


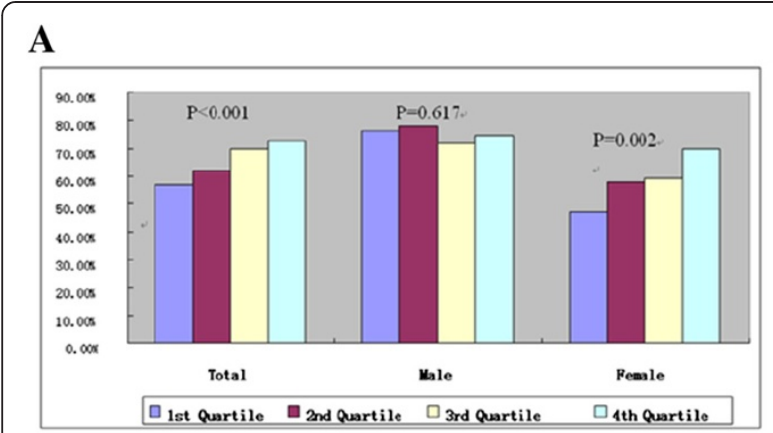

\section{B}

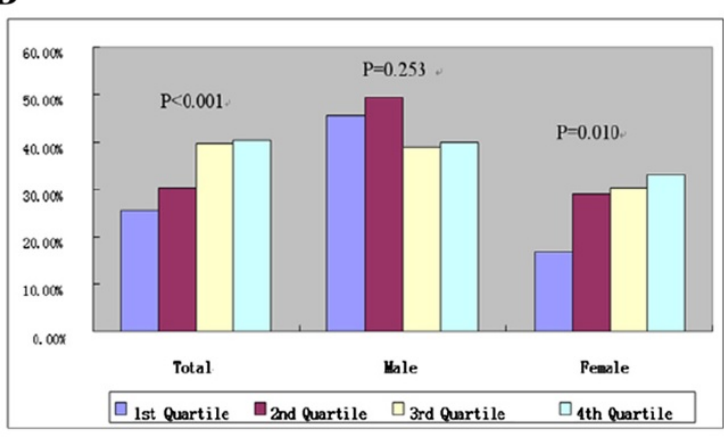

C

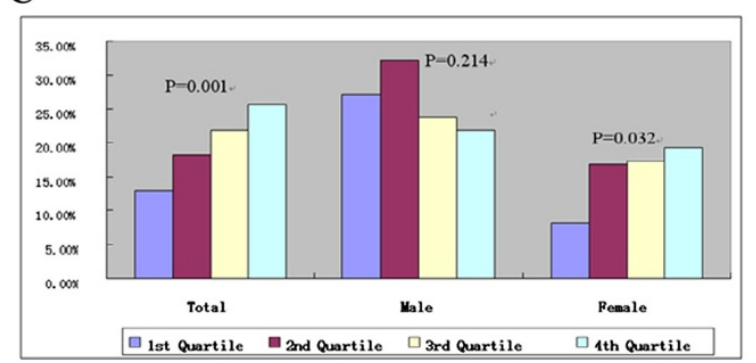

D

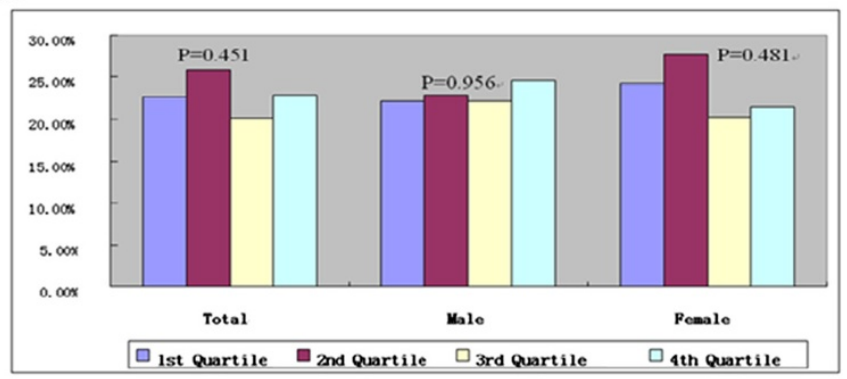

$\mathbf{E}$

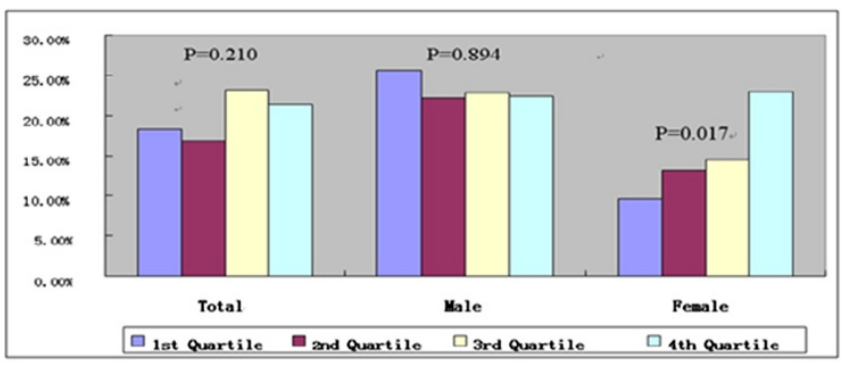

F

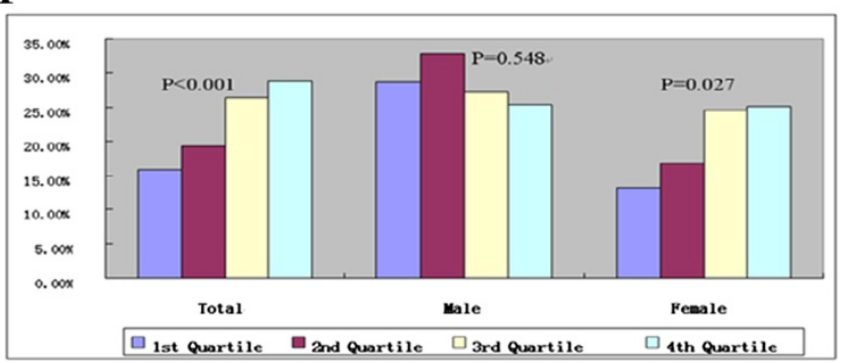

Figure 3 Relationship with anyplaque (A), significant stenosis (B) and severe stenosis (C), single vessel lesion (D), double vessel lesion $(\mathrm{E})$ and triple vessel/left main artery lesion (F) across quartile of serum uric acid levels across quartile of serum uric acid levels.

vs $46.4 \%$ vs $41.0 \%$ vs $35.9 \%, P=0.006$; women: $75.7 \%$ vs $72.3 \%$ vs $60.9 \%$ vs $51.8 \%, P<0.001$, Figure $4 \mathrm{~A})$. The increasing quartiles of UA were significantly associated with a CACS $>10$ in total $(30.6 \%$ vs $32.5 \%$ vs $41.7 \%$ vs $44.5 \%, P=0.001)$ and in women $(22.1 \%$ vs $24.1 \%$ vs $31.2 \%$ vs $41.0 \%, \mathrm{p}=0.002$, Figure $4 \mathrm{~B}$.). As UA levels increased in women, the proportion of mixed plaques significantly increased $(21.7 \%$ vs $22.7 \%$ vs $24.5 \%$ vs $30.5 \%$, $P=0.022$, Figure 5C).

\section{Univariate and multivariate logistic regression models}

In multivariate analysis for men and women combined, age, male sex, smoking, hypertension, and diabetes mellitus (DM) were significantly associated with significant stenosis, multivessel disease, and a high CACS (all p < 0.001). HDL-C level was only significantly associated with significant stenosis and multivessel disease (both $P \leq 0.002$ ). UA was the strongest predictor for significant stenosis, multivessel disease, and a high CACS in univariate analysis (all $P<0.001$ ), but this association was not apparent after adjustment (Table 2).

The same analyses were performed for men and women (Tables 3 and 4). For men, significant predictors of significant stenosis were age, hypertension, and HDL$\mathrm{C}$ levels after adjustment (all $\mathrm{p}<0.05$ ). Significant predictors of multivessel disease were age and hypertension after adjustment (both $P<0.05$ ). The strongest risk factors for a high CACS were age and hypertension after adjustment (both $P<0.05$, Table 3 ). For women, significant predictors of stenosis were age, hypertension, DM, and UA after adjustment (all $P<0.05$ ). Significant predictors of multivessel disease were age, hypertension, DM, and UA after adjustment (all $P<0.05$ ). The strongest risk factors for a high CACS were age, hypertension, 


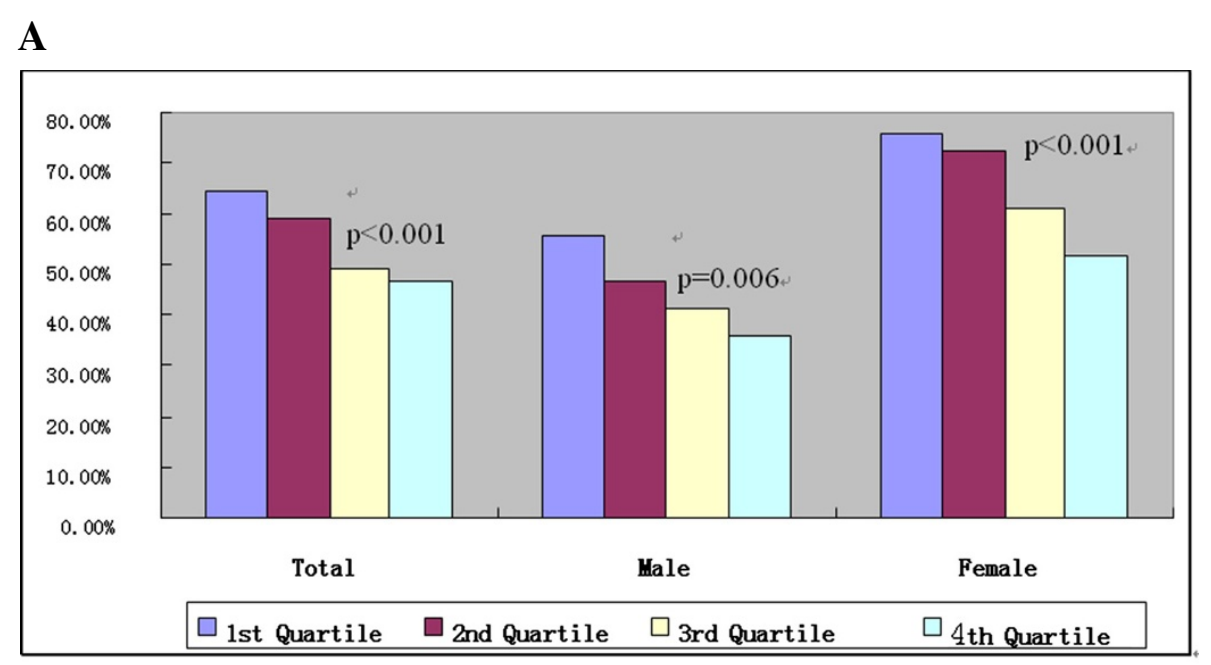

$\mathbf{B}$

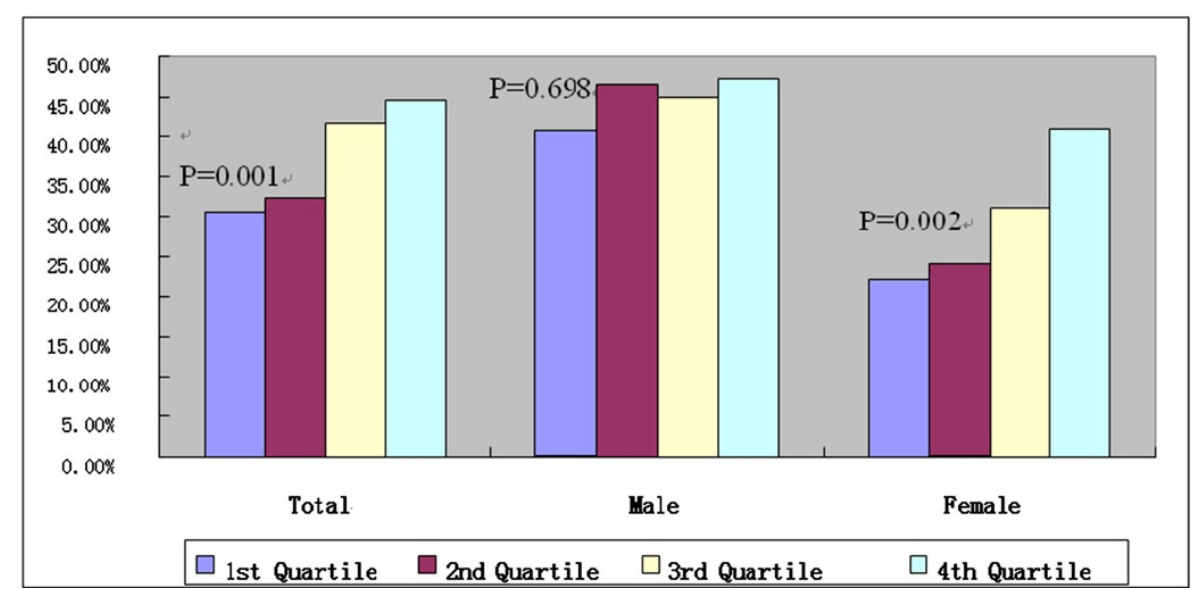

Figure 4 Prevalence of coronary artery calcium score $=0$ across quartile of serum uric acid levels (A), prevalence of coronary artery calcium score $>10$ across quartile of serum uric acid levels (B).

DM, and HDL-C levels after adjustment (all $P<0.05$, Table 4).

In total, significant predictors of significant stenosis were age, male sex, smoking, hypertension, and DM after adjustment (all $P<0.05$ ). Significant predictors of non-calcified plaques were age, smoking, hypertension, DM, and HDL-C levels after adjustment (all $P<0.05$ ). After adjustment, the strongest risk factors for mixed plaques were age, male sex, smoking, hypertension, DM, and UA (all $P<0.05$, Table 5). For men, significant predictors of calcified plaques were age, hypertension, DM, and HDL-C after adjustment (all $P<0.05$ ). The strongest risk factors for non-calcified plaques were age, smoking, DM, and HDL-C in univariate and multivariate analysis (all $P<0.05$ ). The strongest risk factors for a high CACS were age, smoking, and HDL-C levels after adjustment (all $P<0.05$, Table 6). For women, significant predictors of calcified plaques were age, hypertension, and DM after adjustment (all $P<0.05$ ). Significant predictors of non-calcified plaques were age, hypertension, and HDL-C after adjustment (all $P<0.05$ ). Age, hypertension, DM, and UA were significantly associated with mixed plaques in univariate and multivariate analysis (all $P<0.05$, Table 7 ).

\section{Discussion}

In this study of individuals with suspected CAD using 256-detector-row CCTA, we analysed the association of UA with coronary atherosclerosis. We found the following findings. 1) Overall and in women, the prevalence of coronary atherosclerosis, severe stenosis, and triple-vessel/ left main artery lesions of plaques were significantly increased with increasing quartiles of UA. UA was significantly associated with double-vessel lesions in women. 2) 


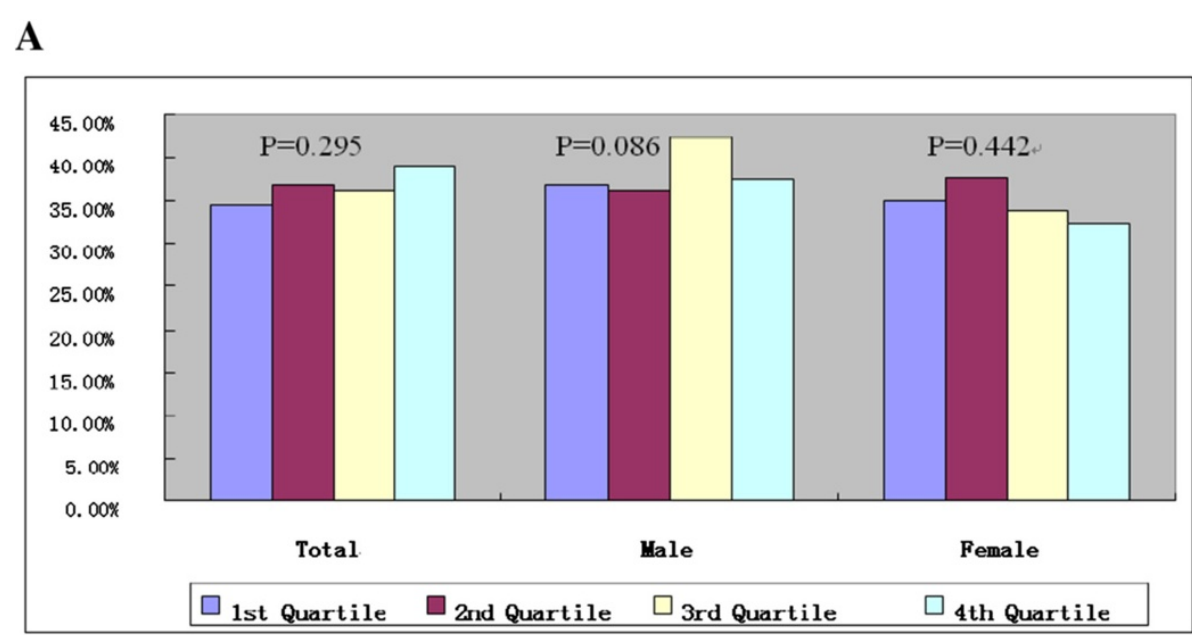

\section{B}

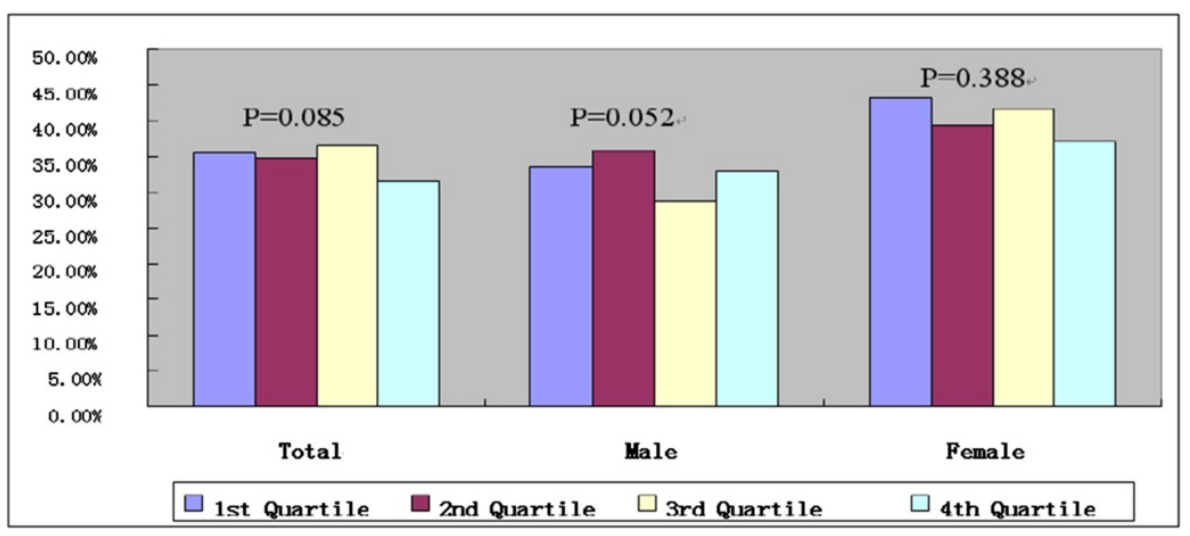

C

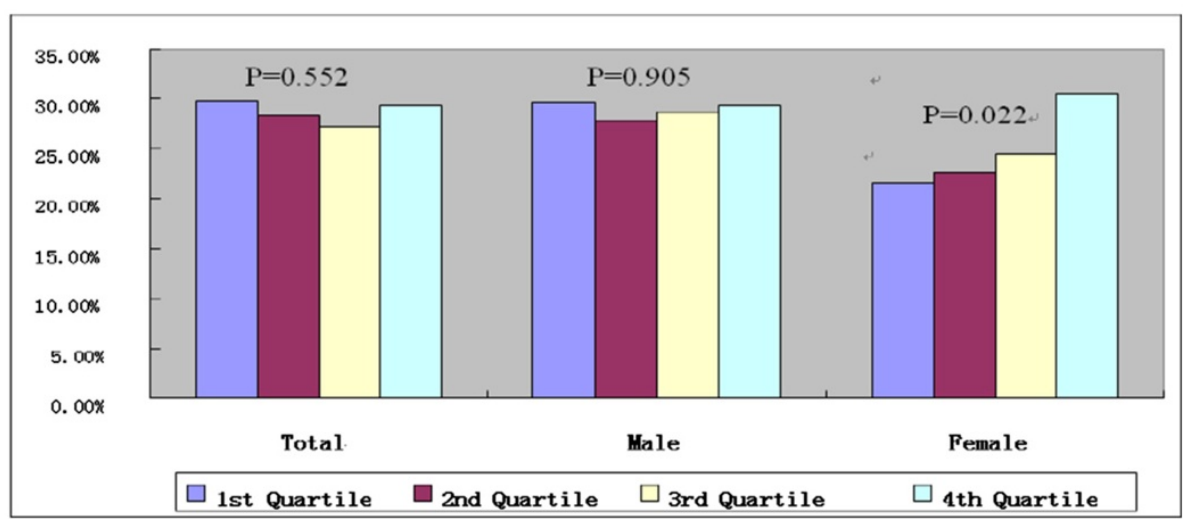

Figure 5 Relationship with (A) calcified, noncalcified (B) and mixed (C) across quartile of serum uric acid levels.

UA was significantly negatively correlated with the proportion of a CACS of 0 , but was positively correlated with the proportion of a CACS $>10$ overall and in women. The incidence of mixed plaques was significantly increased only in women with elevated UA levels. 3) After adjustment, only UA was a significant predictor of significant stenosis, multivessel disease, and mixed plaques in women. UA was not significantly associated with coronary atherosclerosis in men.

Coronary artery angiography (CAG) is the gold standard for diagnosing CAD, but CAG cannot determine the characteristics of plaques. CAG would be difficult to use 
Table 2 Univariate and multivariate logistic regression models for variables of total population associated with coronary artery lesions and high CACS ( $=1116$ )

\begin{tabular}{|c|c|c|c|c|c|c|c|c|c|c|c|c|}
\hline \multirow{3}{*}{$\begin{array}{l}\text { V variable } \\
\text { Variable }\end{array}$} & \multicolumn{4}{|c|}{ Significant stenosis (>50\%) } & \multicolumn{4}{|c|}{ Multivessel disease } & \multicolumn{4}{|c|}{ High CACS (CACS > 100) } \\
\hline & \multicolumn{2}{|l|}{ Univariate } & \multicolumn{2}{|l|}{ Multivariate } & \multicolumn{2}{|c|}{ Univariate } & \multicolumn{2}{|l|}{ Multivariate } & \multicolumn{2}{|c|}{ Univariate } & \multicolumn{2}{|l|}{ Multivariate } \\
\hline & OR $(95 \% \mathrm{Cl})$ & $\mathbf{P}$ & OR $(95 \% \mathrm{Cl})$ & $\mathbf{P}$ & OR $(95 \% \mathrm{Cl})$ & $\mathbf{P}$ & OR $(95 \% \mathrm{Cl})$ & $\mathbf{P}$ & OR $(95 \% \mathrm{Cl})$ & $\mathbf{P}$ & OR $(95 \% \mathrm{Cl})$ & $\mathbf{P}$ \\
\hline Age & 1.059 (1.046-1.073) & $<0.001$ & $1.070(1.054-1.086)$ & $<0.001$ & $1.066(1.052-1.081)$ & $<0.001$ & $1.079(1.062-1.096)$ & $<0.001$ & $1.078(1.061-1.095)$ & $<0.001$ & $1.089(1.070-1.108)$ & $<0.001$ \\
\hline Male & $2.364(1.830-3.054)$ & $<0.001$ & $2.208(1.579-3.089)$ & $<0.001$ & $2.365(1.815-3.083)$ & $<0.001$ & $2.320(1.637-3.288)$ & $<0.001$ & $2.244(1.653-3.047)$ & $<0.001$ & $2.190(1.486-3.226)$ & $<0.001$ \\
\hline BMI & $1.028(0.997-1.060)$ & 0.082 & $1.010(0.975-1.047)$ & 0.568 & $1.027(0.996-1.060)$ & 0.092 & 1.010. (0.974-1.047) & 0.597 & $1.017(0.982-1.052)$ & 0.345 & & \\
\hline Smoking & $1.789(1.355-2.364)$ & $<0.001$ & $1.835(1.306-2.578)$ & $<0.001$ & $1.739(1.308-2.310)$ & $<0.001$ & $1.866(1.313-2.653)$ & 0.001 & $1.854(1.352-2.542)$ & $<0.001$ & $2.294(1.550-3.395)$ & $<0.001$ \\
\hline $\begin{array}{l}\text { Family history } \\
\text { of CAD }\end{array}$ & 1.076 (0.690-1.678) & 0.746 & 一一一一一一 & - - & $1.105(0.701-1.742)$ & 0.666 & & -— & $1.012(0.598-1.714)$ & 0.964 & & \\
\hline Hypertension & $2.187(1.700-2.815)$ & $<0.001$ & $1.856(1.394-2.471)$ & $<0.001$ & $2.275(1.754-2.950)$ & $<0.001$ & $1.914(1.421-2.577)$ & $<0.001$ & $2.377(1.764-3.203)$ & $<0.001$ & $1.939(1.388-2.709)$ & $<0.001$ \\
\hline DM & $2.467(1.788-3.403)$ & $<0.001$ & $1.825(1.278-2.604)$ & $<0.001$ & $2.823(2.041-3.905)$ & $<0.001$ & $2.153(1.501-3.089)$ & $<0.001$ & $2.403(1.694-3.408)$ & $<0.001$ & $1.983(1.344-2.927)$ & 0.001 \\
\hline LDL-C & $0.876(0.758-1.012)$ & 0.072 & $1.156(0.812-1.645)$ & 0.421 & $0.913(0.787-1.059)$ & 0.228 & & & $1.061(0.900-1.251)$ & 0.482 & & \\
\hline HDL-C & $0.331(0.202-0.477)$ & $<0.001$ & $0.456(0.276-0.755)$ & 0.002 & $0.311(0.202-0.477)$ & $<0.001$ & $0.428(0.256-0.717)$ & 0.001 & $0.632(0.396-1.008)$ & 0.054 & $0.907(0.559-1.471)$ & 0.691 \\
\hline TC & $1.802(1.724-1.929)$ & 0.002 & $0.875(0.641-1.194)$ & 0.399 & $1.845(1.744-1.959)$ & 0.009 & $1.022(0.885-1.181)$ & 0.765 & $0.943(0.819-1.086)$ & 0.415 & & \\
\hline TG & $0.983(0.895-1.081)$ & 0.726 & & 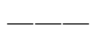 & $0.992(0.901-1.092)$ & 0.863 & & 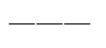 & $0.889(0.771-1.024)$ & 0.145 & & -—— \\
\hline UA & $1.003(1.002-1.004)$ & $<0.001$ & $1.001(0.999-1.002)$ & 0.535 & $1.003(1.002-1.004)$ & $<0.001$ & $1.001(0.999-1.002)$ & 0.564 & $1.003(1.001-1.005)$ & $<0.001$ & 1.001 (0.999-1.003) & 0.205 \\
\hline Creatinine & $0.980(0.857-1.018)$ & 0.120 & -二-二-二- & -— & $1.240(0.957-1.417)$ & 0.258 & -—— & -—— & $0.760(0.526-1.013)$ & 0.173 & - & -— \\
\hline
\end{tabular}

Multivessel disease was defined as the present of stenosis of more than $50 \%$ in at lease two vessels.

Risk factors with $\mathrm{p}<0.10$ in unvariate adjusted simultaneously. Blanks indicate variables not adjusted in multivariate analyses. 
Table 3 Univariate and multivariate logistic regression models for variables of male associated with coronary artery lesions and high CACS ( $=566$ )

\begin{tabular}{|c|c|c|c|c|c|c|c|c|c|c|c|c|}
\hline \multirow{3}{*}{$\begin{array}{l}\text { V variable } \\
\text { Variable }\end{array}$} & \multicolumn{4}{|c|}{ Significant stenosis (>50\%) } & \multicolumn{4}{|c|}{ Multivessel disease } & \multicolumn{4}{|c|}{ High CACS (CACS > 100) } \\
\hline & \multicolumn{2}{|c|}{ Univariate } & \multicolumn{2}{|l|}{ Multivariate } & \multicolumn{2}{|c|}{ Univariate } & \multicolumn{2}{|l|}{ Multivariate } & \multicolumn{2}{|c|}{ Univariate } & \multicolumn{2}{|l|}{ Multivariate } \\
\hline & OR $(95 \% \mathrm{Cl})$ & $\mathbf{P}$ & OR $(95 \% \mathrm{Cl})$ & $\mathbf{P}$ & OR $(95 \% \mathrm{Cl})$ & $\mathbf{P}$ & OR $(95 \% \mathrm{Cl})$ & $\mathbf{P}$ & OR $(95 \% \mathrm{Cl})$ & $\mathbf{P}$ & $\mathrm{OR}(95 \% \mathrm{Cl})$ & $\mathbf{P}$ \\
\hline Age & $1.056(1.039-1.073)$ & $<0.001$ & $1.052(1.034-1.070)$ & $<0.001$ & $1.060(1.043-1.077)$ & $<0.001$ & $1.055(1.037-1.073)$ & $<0.001$ & $1.077(1.057-1.098)$ & $<0.001$ & $1.071(1.050-1.093)$ & $<0.001$ \\
\hline BMI & $0.978(0.937) 1.020$ & 0.296 & 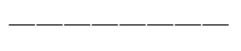 & -—- & $0.979(0.938-1.022)$ & 0.329 & 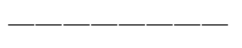 & 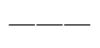 & $0.955(0.906-1.006)$ & 0.084 & $0.996(0.945-1.051)$ & 0.894 \\
\hline Smoking & $1.245(0.889-1.743)$ & 0.203 & & -—- & $1.298(0.926-1.820)$ & 0.131 & & ——— & $1.305(0.896-1.900)$ & 0.166 & & \\
\hline $\begin{array}{l}\text { Family history } \\
\text { of CAD }\end{array}$ & $1.274(0.684-2.375)$ & 0.446 & & -— & $1.501(0.805-2.798)$ & 0.201 & & -—— & $1.080(0.539-2.162)$ & 0.828 & & \\
\hline Hypertension & $1.811(1.291-2.541)$ & 0.001 & $1.579(1.098-2.270)$ & 0.014 & $1.734(1.235-2.434)$ & 0.001 & $1.462(1.016-2.104)$ & 0.041 & $2.044(1.400-2.983)$ & $<0.001$ & $1.731(1.138-2.634)$ & 0.010 \\
\hline DM & $1.608(1.045-2.473)$ & 0.031 & $1.368(0.862-2.172)$ & 0.184 & $1.727(1.123-2.658)$ & 0.013 & $1.579(0.998-2.499)$ & 0.051 & $1.585(1.001-2.511)$ & 0.049 & $1.581(0.952-2.624)$ & 0.077 \\
\hline LDL-C & $0.856(0.694-1.054)$ & 0.143 & -———- & -—— & $0.919(0.746-1.133)$ & 0.430 & & & $0.986(0.782-1.243)$ & 0.902 & & \\
\hline HDL-C & $0.575(0.317-1.044)$ & 0.069 & $0.485(0.236-0.995)$ & 0.048 & $0.635(0.350-1.152)$ & 0.135 & 一ー一一一一—— & 一一一 & $0.435(0.072-0.797)$ & 0.030 & $1.676(0.813-3.453)$ & 0.162 \\
\hline TC & $1.764(1.641) 1.911$ & 0.003 & $0.880(0.712-1.088)$ & 0.239 & $1.817(1.688-1.971)$ & 0.022 & $0.875(0.721-1.062)$ & 0.176 & $0.891(0.738-1.075)$ & 0.229 & & \\
\hline TG & 1.868 (1.759-1.994) & 0.041 & $0.939(0.797-1.106)$ & 0.451 & $0.874(0.763-1.001)$ & 0.051 & $0.998(0.864-1.152)$ & 0.973 & $1.754(1.616-1.924)$ & 0.006 & $0.926(0.754-1.137)$ & 0.464 \\
\hline UA & $1.000(0.998-1.002)$ & 0.923 & & —— & $1.000(0.998-1.002)$ & 0.716 & & -5 & $1.000(0.998-1.002)$ & 0.960 & & \\
\hline Creatinine & $1.403(0.549-3.073)$ & 0.613 & -—————- & -—— & $0.923(0.238-1.997)$ & 0.588 & & -—— & $0.574(0.185-1.236)$ & 0.339 & & -3 \\
\hline
\end{tabular}

Multivessel disease was defined as the present of stenosis of more than $50 \%$ in at lease two vessels.

Risk factors with $\mathrm{p}<0.10$ in unvariate adjusted simultaneously. Blanks indicate variables not adjusted in multivariate analyses. 
Table 4 Univariate and multivariate logistic regression models for variables of female associated with coronary artery lesions and high CACS ( $=550$ )

\begin{tabular}{|c|c|c|c|c|c|c|c|c|c|c|c|c|}
\hline \multirow{3}{*}{$\begin{array}{l}\text { V variable } \\
\text { Variable }\end{array}$} & \multicolumn{4}{|c|}{ Significant stenosis ( $>50 \%)$} & \multicolumn{4}{|c|}{ Multivessel disease } & \multicolumn{4}{|c|}{ High CACS (CACS > 100) } \\
\hline & \multicolumn{2}{|c|}{ Univariate } & \multicolumn{2}{|l|}{ Multivariate } & \multicolumn{2}{|c|}{ Univariate } & \multicolumn{2}{|l|}{ Multivariate } & \multicolumn{2}{|c|}{ Univariate } & \multicolumn{2}{|l|}{ Multivariate } \\
\hline & OR $(95 \% \mathrm{Cl})$ & $\mathbf{P}$ & OR $(95 \% \mathrm{Cl})$ & $\mathbf{P}$ & OR $(95 \% \mathrm{Cl})$ & $\mathbf{P}$ & OR $(95 \% \mathrm{Cl})$ & $\mathbf{P}$ & OR $(95 \% \mathrm{Cl})$ & $\mathbf{P}$ & OR $(95 \% \mathrm{Cl})$ & $\mathbf{P}$ \\
\hline Age & $1.096(1.069-1.123)$ & $<0.001$ & $1.084(1.055-1.113)$ & $<0.001$ & $1.092(1.068-1.117)$ & $<0.001$ & $1.075(1.049-1.101)$ & $<0.001$ & $1.100(1.068-1.133)$ & $<0.001$ & $1.085(1.049-1.122)$ & $<0.001$ \\
\hline BMI & $1.071(1.013-1.132)$ & 0.015 & $1.024(0.985-1.103)$ & 0.151 & $1.073(1.018-1.132)$ & 0.009 & $1.036(0.980-1.095)$ & 0.216 & $1.077(1.012-1.147)$ & 0.020 & $1.060(0.998-1.126)$ & 0.060 \\
\hline Smoking & $1.434(0.723-2.845)$ & 0.303 & & -—- & $1.317(0.692-2.504)$ & 0.402 & & & $1.795(0.822-3.918)$ & 0.142 & & \\
\hline $\begin{array}{l}\text { Family history } \\
\text { of CAD }\end{array}$ & $0.978(0.496-1.932)$ & 0.950 & & -— & $0.716(0.376-1.363)$ & 0.309 & & - - & $1.017(0.440-2.351)$ & 0.969 & & \\
\hline Hypertension & $3.144(2.096-4.718)$ & $<0.001$ & $2.229(1.420-3.500)$ & $<0.001$ & $3.689(2.550-5.336)$ & $<0.001$ & $2.618(1.735-3.950)$ & $<0.001$ & $3.385(2.017-5.680)$ & $<0.001$ & $2.162(1.216-3.843)$ & 0.009 \\
\hline DM & $4.144(2.536-6.772)$ & $<0.001$ & $2.669(1.554-4.584)$ & $<0.001$ & $4.301(2.620-7.061)$ & $<0.001$ & $2.913(1.682-5.044)$ & $<0.001$ & $4.286(2.476-7.418)$ & $<0.001$ & $2.958(1.582-5.531)$ & 0.001 \\
\hline LDL-C & $1.049(0.851-1.293)$ & 0.652 & & -—- & $1.044(0.863-1.263)$ & 0.654 & & -—— & $1.381(1.078-1.769)$ & 0.011 & $2.090(0.868-5.036)$ & 0.100 \\
\hline HDL-C & $0.309(0.158-0.602)$ & $<0.001$ & $0.462(0.219-0.977)$ & 0.430 & $0.344(0.192-0.618)$ & $<0.001$ & $0.642(0.336-1.227)$ & 0.180 & $0.265(0.112-0.629)$ & 0.003 & $0.327(0.118-0.908)$ & 0.032 \\
\hline TC & $1.056(0.878-1.271)$ & 0.561 & -— & -—— & $1.054(0.890-1.247)$ & 0.544 & - & -—— & $1.236(0.991-1.541)$ & 0.060 & $0.776(0.346-1.741)$ & 0.776 \\
\hline TG & $1.190(0.994-1.424)$ & 0.058 & $1.043(0.852-1.276)$ & 0.684 & $1.338(1.103-1.623)$ & 0.003 & $1.131(0.913-1.401)$ & 0.259 & $1.094(0.897-1.334)$ & 0.374 & —— & -—— \\
\hline UA & $1.214(1.101-1.407)$ & 0.002 & $1.004(1.001-1.007)$ & 0.011 & 1.115 (1.103-1.134) & $<0.001$ & $\begin{array}{c}11.131(0.913-1.401) \\
1.003(1.000-1.006)\end{array}$ & $<0.001$ & $1.005(1.001-1.008)$ & 0.004 & $1.001(0.997-1.004)$ & 0.793 \\
\hline Creatinine & $1.835(0.511-3.164)$ & 0.535 & -——--——- & -—— & $0.714(0.138-2.227)$ & 0.407 & -——-——-— & -—— & $0.925(0.238-2.764)$ & 0.868 & & - \\
\hline
\end{tabular}

Multivessel disease was defined as the present of stenosis of more than $50 \%$ in at lease two vessels.

Risk factors with $\mathrm{p}<0.10$ in unvariate adjusted simultaneously. Blanks indicate variables not adjusted in multivariate analyses. 
Table 5 Univariate and multivariate logistic regression models for variables of total population associated with calcified, non-calcified and mixed plaque $(n=1116)$

\begin{tabular}{|c|c|c|c|c|c|c|c|c|c|c|c|c|}
\hline \multirow{3}{*}{$\begin{array}{l}\text { V variable } \\
\text { Variable }\end{array}$} & \multicolumn{4}{|c|}{ Calcified plaque } & \multicolumn{4}{|c|}{ Non-calcified plaque } & \multicolumn{4}{|c|}{ Mixed plaque } \\
\hline & \multicolumn{2}{|l|}{ Univariate } & \multicolumn{2}{|l|}{ Multivariate } & \multicolumn{2}{|l|}{ Univariate } & \multicolumn{2}{|l|}{ Multivariate } & \multicolumn{2}{|l|}{ Univariate } & \multicolumn{2}{|c|}{ Multivariate } \\
\hline & OR $(95 \% \mathrm{Cl})$ & $\mathbf{P}$ & OR $(95 \% \mathrm{Cl})$ & $\mathbf{P}$ & OR $(95 \% \mathrm{Cl})$ & $\mathbf{P}$ & OR $(95 \% \mathrm{Cl})$ & $\mathbf{P}$ & OR $(95 \% \mathrm{Cl})$ & $\mathbf{P}$ & OR $(95 \% \mathrm{Cl})$ & $\mathbf{P}$ \\
\hline Age & $1.071(1.057-1.086)$ & $<0.001$ & $1.052(1.023-1.081)$ & $<0.001$ & $1.028(1.016-1.040)$ & $<0.001$ & $1.034(1.021-1.047)$ & $<0.001$ & $1.056(1.042-1.069)$ & $<0.001$ & $1.066(1.051-1.081)$ & $<0.001$ \\
\hline Male & $2.022(1.579-2.590)$ & $<0.001$ & $2.282(1.662-3.132)$ & $<0.001$ & $1.666(1.315-2.111)$ & $<0.001$ & $1.249(0.925-1.686)$ & $<0.147$ & $2.328(1.806-3.000)$ & $<0.001$ & $2.002(1.445-2.774)$ & $<0.001$ \\
\hline BMI & $1.017(0.987-1.048)$ & 0.276 & -_——_ & - - - & $1.045(1.012-1.080)$ & 0.008 & $1.023(0.988-1.058)$ & 0.202 & $1.021(0.991-1.053)$ & 0.173 & —— & -—— \\
\hline Smoking & $1.428(1.084-1.881)$ & 0.011 & $1.224(1.036-1.467)$ & 0.043 & $1.707(1.297-2.246)$ & $<0.001$ & $1.729(1.258-2.376)$ & 0.001 & $2.171(1.645-2.864)$ & $<0.001$ & $2.336(1.669-3.270)$ & $<0.001$ \\
\hline $\begin{array}{l}\text { Family history } \\
\text { of CAD }\end{array}$ & $0.825(0.527-1.293)$ & 0.402 & 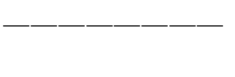 & ——— & $1.183(0.773-1.810)$ & 0.438 & & ——— & $1.022(0.655-1.592)$ & 0.925 & & \\
\hline Hypertension & $2.180(1.703-2.792)$ & $<0.001$ & $1.832(1.393-2.409)$ & $<0.001$ & $1.579(1.244-2.005)$ & $<0.001$ & $1.319(1.017-1.711)$ & 0.037 & $2.090(1.627-2.683)$ & $<0.001$ & $1.810(1.367-2.395)$ & $<0.001$ \\
\hline DM & $2.538(1.839-3.503)$ & $<0.001$ & $2.065(1.524-2.265)$ & $<0.001$ & $1.924(1.390-2.665)$ & $<0.001$ & $1.510(1.070-2.130)$ & 0.019 & $2.386(1.731-3.291)$ & $<0.001$ & $1.917(1.346-2.730)$ & $<0.001$ \\
\hline LDL-C & $1.669(0.930-1.227)$ & 0.348 & & - & $0.945(0.826-1.082)$ & 0.945 & - - - & ——— & $0.981(0.852-1.129)$ & 0.786 & -— & - - \\
\hline HDL-C & $0.851(0.601-1.206)$ & 0.365 & & & $0.347(0.235-0.512)$ & $<0.001$ & $0.493(0.322-0.757)$ & 0.001 & $0.504(0.338-0.752)$ & 0.001 & $0.763(0.501-1.164)$ & 0.210 \\
\hline TC & $1.009(0.899-1.132)$ & 0.878 & & & $0.930(0.831-1.041)$ & 0.208 & ———————— & ——— & $0.905(0.803-1.020)$ & 0.103 & & \\
\hline TG & $0.998(0.912-1.093)$ & 0.969 & -——-—— & -—— & $1.113(1.009-1.228)$ & 0.033 & $1.032(0.933-1.140)$ & 0.542 & $1.044(0.954-1.141)$ & 0.348 & & -—— \\
\hline UA & $1.003(1.001-1.004)$ & $<0.001$ & $1.002(0.998-1.003)$ & 0.244 & $1.003(1.002-1.004)$ & $<0.001$ & $1.001(0.999-1.002)$ & 0.113 & $1.004(1.002-1.005)$ & $<0.001$ & $1.002(1.001-1.003)$ & 0.046 \\
\hline Creatinine & $1 . .020(1.005-1.036)$ & 0.005 & $0.823(0.422-1.329)$ & 0.201 & $1.033(0.894-1.086)$ & 0.165 & -——-—-—- & -—— & $1.157(0.811-1.425)$ & 0.148 & -— & - \\
\hline
\end{tabular}

Risk factors with $\mathrm{p}<0.10$ in unvariate adjusted simultaneously. Blanks indicate variables not adjusted in multivariate analyses. 
Table 6 Univariate and multivariate logistic regression models for variables of male associated with calcified, non-calcified and mixed plaque ( $\mathbf{n}=566$ )

\begin{tabular}{|c|c|c|c|c|c|c|c|c|c|c|c|c|}
\hline \multirow{3}{*}{$\begin{array}{l}\text { V variable } \\
\text { Variable }\end{array}$} & \multicolumn{4}{|c|}{ Calcified plaque } & \multicolumn{4}{|c|}{ Non-calcified plaque } & \multicolumn{4}{|c|}{ Mixed plaque } \\
\hline & \multicolumn{2}{|l|}{ Univariate } & \multicolumn{2}{|l|}{ Multivariate } & \multicolumn{2}{|l|}{ Univariate } & \multicolumn{2}{|l|}{ Multivariate } & \multicolumn{2}{|l|}{ Univariate } & \multicolumn{2}{|l|}{ Multivariate } \\
\hline & OR $(95 \% \mathrm{Cl})$ & $\mathbf{P}$ & OR $(95 \% \mathrm{Cl})$ & $\mathbf{P}$ & OR $(95 \% \mathrm{Cl})$ & $\mathbf{P}$ & OR $(95 \% \mathrm{Cl})$ & $\mathbf{P}$ & OR $(95 \% \mathrm{Cl})$ & $\mathbf{P}$ & OR $(95 \% \mathrm{Cl})$ & $\mathbf{P}$ \\
\hline Age & $1.069(1.051-1.088)$ & $<0.001$ & $1.068(1.049-1.087)$ & $<0.001$ & $1.018(1.003-1.033)$ & 0.016 & $1.024(1.008-1.040)$ & 0.003 & $1.048(1.032-1.064)$ & $<0.001$ & $1.056(1.038-1.075)$ & $<0.001$ \\
\hline BMI & $0.979(0.939-1.021)$ & 0.315 & $-\ldots$ & - - - & $1.009(0.969-1.051)$ & 0.664 & -- - & ——— & $0.986(0.946-1.027)$ & 0.500 & -—— & -—— \\
\hline Smoking & $1.041(0.745-1.456)$ & 0.812 & & 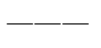 & $1.484(1.058-2.082)$ & 0.022 & $1.709(1.194-2.447)$ & 0.003 & $1.597(1.140-2.237)$ & 0.007 & $2.258(1.548-3.294)$ & $<0.001$ \\
\hline $\begin{array}{l}\text { Family history } \\
\text { of CAD }\end{array}$ & $0.771(0.409-1.455)$ & 0.422 & 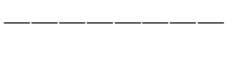 & -—— & $1.409(0.742-2.678)$ & 0.295 & & . & $1.488(0.797-2.776)$ & 0.212 & & \\
\hline Hypertension & $1.745(1.246-2.445)$ & 0.001 & $1.522(1.051-2.204)$ & 0.026 & $1.291(0.922-1.808)$ & 0.137 & & & $1.533(1.095-2.146)$ & 0.013 & $1.411(0.978-2.036)$ & 0.066 \\
\hline DM & $1.672(1.086-2.575)$ & 0.020 & $1.646(1.027-2.637)$ & 0.038 & $1.902(1.209-2.991)$ & 0.005 & $1.660(1.043-2.642)$ & 0.033 & $1.763(1.144-2.716)$ & 0.010 & $1.501(0.942-2.393)$ & 0.088 \\
\hline LDL-C & $1.167(0.948-1.436)$ & 0.145 & -—— & ——— & $0.932(0.757-1.146)$ & 0.503 & -— & -—- & $0.954(0.775-1.173)$ & 0.655 & -— & 一— \\
\hline HDL-C & $0.219(0.094-0.344)$ & 0.007 & $0.358(0.169-0.547)$ & 0.016 & $0.489(0.271-0.882)$ & 0.018 & $0.455(0.248-0.832)$ & 0.011 & $0.478(0.261-0.874)$ & 0.017 & $0.387(0.200-0.749)$ & 0.005 \\
\hline TC & $1.058(0.900-1.244)$ & 0.496 & 一-———— & ——- & $0.970(0.825-1.141)$ & 0.717 & & 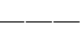 & $0.851(0.719-1.006)$ & 0.059 & $0.946(0.785-1.140)$ & 0.561 \\
\hline TG & $0.893(0.787-1.013)$ & 0.080 & $1.046(0.925-1.182)$ & 0.476 & $1.092(0.967-1.233)$ & 0.158 & & & $0.990(0.890-1.102)$ & 0.855 & & \\
\hline UA & 0.999 (0.998-1.001) & 0.601 & 一一一 & 一一一 & 1.001 (0.999-1.003) & 0.232 & & & 1.001 (0.999-1.003) & 0.498 & & \\
\hline Creatinine & $1.061(0.777-1.321)$ & 0.543 & -———-——- & - - & $1.980(0.860-4.522)$ & 0.126 & -— & ——— & $0.631(0.483-1.376)$ & 0.194 & & \\
\hline
\end{tabular}

Risk factors with $\mathrm{p}<0.10$ in unvariate adjusted simultaneously. Blanks indicate variables not adjusted in multivariate analyses. 
Table 7 Univariate and multivariate logistic regression models for variables of female associated with calcified, non-calcified and mixed plaque ( $\mathbf{n}=550$ )

\begin{tabular}{|c|c|c|c|c|c|c|c|c|c|c|c|c|}
\hline \multirow{3}{*}{$\begin{array}{l}\text { V variable } \\
\text { Variable }\end{array}$} & \multicolumn{4}{|c|}{ Calcified plaque } & \multicolumn{4}{|c|}{ Non-calcified plaque } & \multicolumn{4}{|c|}{ Mixed plaque } \\
\hline & \multicolumn{2}{|l|}{ Univariate } & \multicolumn{2}{|l|}{ Multivariate } & \multicolumn{2}{|c|}{ Univariate } & \multicolumn{2}{|l|}{ Multivariate } & \multicolumn{2}{|l|}{ Univariate } & \multicolumn{2}{|c|}{ Multivariate } \\
\hline & OR $(95 \% \mathrm{Cl})$ & $\mathbf{P}$ & OR $(95 \% \mathrm{Cl})$ & $\mathbf{P}$ & OR $(95 \% \mathrm{Cl})$ & $\mathbf{P}$ & OR $(95 \% \mathrm{Cl})$ & $\mathbf{P}$ & OR $(95 \% \mathrm{Cl})$ & $\mathbf{P}$ & OR $(95 \% \mathrm{Cl})$ & $\mathbf{P}$ \\
\hline Age & $1.101(1.075-1.127)$ & $<0.001$ & $1.083(1.056-1.111)$ & $<0.001$ & $1.057(1.036-1.078)$ & $<0.001$ & $1.048(1.026-1.070)$ & $<0.001$ & $1.101(1.074-1.128)$ & $<0.001$ & $1.086(1.058-1.115)$ & $<0.001$ \\
\hline BMI & $1.041(0.991-1.093)$ & 0.110 & -—-—--—- & -—- & $1.073(1.018-1.131)$ & 0.008 & $1.047(0.992-1.106)$ & 0.098 & $1.038(0.987-1.090)$ & 0.145 & & \\
\hline Smoking & $1.101(0.557-2.177)$ & 0.782 & & & $1.259(0.670-2.366)$ & 0.474 & & 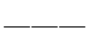 & $1.702(0.877-3.302)$ & 0.116 & & \\
\hline $\begin{array}{l}\text { Family history } \\
\text { of CAD }\end{array}$ & $0.943(0.494-1.800)$ & 0.859 & & -—- & $1.076(0.599-1.933)$ & 0.806 & & ——— & $0.712(0.346-1.464)$ & 0.355 & & \\
\hline Hypertension & $3.078(2.101-4.508)$ & $<0.001$ & $2.216(1.453-3.380)$ & $<0.001$ & $1.991(1.411-2.810)$ & $<0.001$ & $1.506(1.037-2.188)$ & 0.032 & $3.503(2.340-5.244)$ & $<0.001$ & $2.720(1.759-4.206)$ & $<0.001$ \\
\hline DM & $4.093(2.513-6.665)$ & $<0.001$ & $2.845(1.666-4.858)$ & $<0.001$ & $1.868(1.161-3.006)$ & 0.010 & $1.287(0.772-2.146)$ & 0.333 & $3.383(2.076-5.513)$ & $<0.001$ & $2.192(1.281-3.750)$ & 0.004 \\
\hline LDL-C & $1.132(0.930-1.379)$ & 0.216 & & & $1.046(0.870-1.257)$ & 0.633 & -—— & -—- & $1.186(0.967-1.455)$ & 0.101 & & \\
\hline HDL-C & $0.693(0.404-1.188)$ & 0.182 & & & $0.358(0.206-0.622)$ & $<0.001$ & $0.486(0.272-0.870)$ & 0.015 & $0.965(0.596-1.561)$ & 0.884 & & \\
\hline TC & $1.104(0.926-1.315)$ & 0.270 & - - & ——— & $0.982(0.834-1.156)$ & 0.824 & -- - - - - & ——— & $1.157(0.965-1.388)$ & 0.115 & & -— \\
\hline TG & $1.202(1.005-1.438)$ & 0.044 & $1.111(0.919-1.343)$ & 0.277 & $1.111(0.941-1.312)$ & 0.215 & & ——— & $1.112(0.937-1.320)$ & 0.224 & & ——— \\
\hline UA & $1.004(1.002-1.007)$ & 0.001 & $1.001(0.998-1.434)$ & 0.393 & $1.004(1.001-1.006)$ & 0.002 & $1.001(0.998-1.004)$ & 0.500 & $1.004(1.002-1.007)$ & 0.001 & $1.003(0.001-1.006)$ & 0.015 \\
\hline Creatinine & $1.119(0.866-1.288)$ & 0.274 & -二-二-—- & -—- & $1.026(0.687-1.537)$ & 0.253 & 一一一-——- & - - & $0.498(0.173-2.051)$ & 0.341 & - & -—- \\
\hline
\end{tabular}

Risk factors with $\mathrm{p}<0.10$ in unvariate adjusted simultaneously. Blanks indicate variables not adjusted in multivariate analyses. 
as a general screening tool in China because of its highly invasive nature, radiation exposure, and cost. The technique of 256-detector-row CCTA with a shorter scanning time can improve image quality and it has a minimal radiation dose [18]. Use of CCTA in a general routine health evaluation is not discouraged [19]. Therefore, healthy subjects were not included in our study. We enrolled those patients with suspected CAD who needed to have a CCTA examination while undergoing a clinician's assessment. CCTA is a screening tool for assessing individuals of suspected CAD, and is the most feasible and effective method to reflect widespread epidemiology and characteristics of early CAD in China.

Many studies have assessed the association of UA with $\mathrm{CAD}$ and clinical outcome, but the results were still controversial. Kocaman et al. found that UA was an independent predictor of CAD in individuals undergoing CAG [20]. However, the Framingham studies showed that UA was not an independent predictor of CAD and cardiovascular outcomes [21], and similar results were found in the ARIC study [22] and a study by Strask et al. [23]. Carotid intima-media thickness (C-IMT) measured by ultrasonography is widely used as a surrogate marker for atherosclerotic disease and directly associated with increased risk of cardiovascular disease. The higher UA levels were associated with higher C-IMT independent from hypertension, UA levels were independently associated with C-IMT $[24,25]$. But a study shown UA was not significantly associated with C-IMT, UA was associated with Internal carotid artery resistive index in hypertensive women, suggesting that there might gender-related differences in the relationship between UA and vascular damage [26]. In our study, although the prevalence and degree of plaques were significantly increased with UA overall, UA was not a significant independent predictor. Several studies have shown conflicting results on the association between UA and CAD in men and women. Some studies have demonstrated that UA was an independent risk factor for CAD in both sexes [13,14]. A high UA level was found to be an independent predictor for cardiovascular mortality in men [27]. In the LIFE study, the significant association of UA with CAD was found only in women [12]. Cardiovascular death was increased by 1.77 times in men and 3.0 times in women in the upper UA quartile in the NHANES I study [28]. A previous study reported that UA levels only in women were associated with CAD [29]. Our study supported the opinion that UA was an independent predictor for the prevalence and severity of early CAD only in women.

Previous reports have proven the highly predictive value of the CACS on cardiovascular outcomes [30,31]. Some studies have shown a significant relationship between UA and the CACS [32-34], and a recent study showed that UA was an independent factor predictive of the CACS [34]. However, some studies did not support these previous findings $[7,35]$. In our study, we found that UA was significantly associated with the CACS, but UA was not an independent risk factor for a high CACS overall, in men and women. The relationship between UA and CACS, and the concrete mechanisms are still unclear. Few studies have focused on the role of UA in characterization of plaques as shown by CCTA. One study showed that UA was only significantly associated with calcified plaques [36]. In our study, only UA was an independent predictor for mixed plaques overall and in women. The relationship between UA and mixed plaques was likely caused by the female subgroup. Many studies have indicated that mixed plaques might be the biggest risk of plaque rupture, causing acute coronary events $[37,38]$. This may be a rational explanation for why high UA level was associated with an increase in the prevalence of mixed plaques in women, which causes an increase in the incidence of adverse cardiovascular outcomes. However, there are too few studies on the relationship between UA and plaque characteristics, and further studies are required.

UA is a general antioxidant in the body, and a high UA level is suggestive of oxidative stress, endothelial dysfunction, and slow coronary artery flow $[39,40]$. UA promotes vascular smooth muscle proliferation, and upregulates the expression of monocyte chemoattractant protein-1 and platelet-derived growth factor [41]. Endothelial dysfunction is an important step in the development of atherosclerosis. A recent study showed a relationship between UA and endothelial dysfunction of coronary microvasculature only in women [42], which may be a possible explanation for the significant association between UA and CAD in women. The underlying mechanisms behind the significant relationship between $\mathrm{UA}$ and CAD in women but not men remain enigmatic. Our study suggests that UA plays an important role in coronary atherosclerosis in women.

The incidence of cardiovascular events is still high after controlling for traditional risk factors. Our study suggests that UA should be considered as an additional risk factor beyond traditional factors for CAD in women. However, a randomized study still needs to be performed to determine the effect of UA-lowering therapies on cardiovascular prevention. In the GREACE study, decreased UA levels by atorvastatin were independently correlated with a reduced risk of cardiovascular outcomes [43]. The LIFE study showed an association between UA and cardiovascular events in hypertensive women, the unique results may be due in part to the specific feature of reduction of UA by losartan, but it did not mean that the beneficial effects observed with losartan were contributed to decreasing UA levels [12]. The 
relationship between low UA level and cardiovascular benefits is still uncertain. Therefore, further research should be performed to assess the direct relationship between decreasing UA level and cardiovascular benefits.

There were several limitations to our study. All individuals were suspected of having CAD, we mainly want to explore the relationship between UA and early CAD in the study. Patients with known CAD may implement lifestyle modifications and drugs intervention to some extent, the real relationship between UA and CAD may be influenced by those confounding factors, So, we exclude the patients who had known CAD. There was a possible selection bias and this could restrict generalizability of our results to similar care settings. Unmeasured confounders may have affected our results. In our study, almost $90 \%$ of women were post-menopausal and few women received oestrogenreplacement therapy. Therefore, we did not analyse the potential effects of oestrogen on the relationship between UA and CAD. In cross-sectional analysis, we did not find a longitudinal relationship between UA and cardiovascular outcomes. In spite of these limitations, the strong association of UA with early CAD in women had an important clinical significance in prevention and treatment of CAD. Longitudinal studies are required to confirm the association of UA with CAD and cardiovascular outcome.

\section{Conclusion}

UA is a significant predictor of significant stenosis, multivessel disease, and mixed plaques in women. Importantly, our study suggests that UA level may play an important role in the occurrence and development of coronary atherosclerosis in women but not men.

\section{Abbreviations \\ UA: Uric acid; CAD: Coronary artery disease; CCTA: Coronary computed tomography angiography; CACS: Calcium score; BMI: Body mass index; TC: Total cholesterol; TG: Triglyceride; HDL-C: High-density lipoprotein cholesterol; LDL-C: Low-density lipoprotein cholesterol; CAG: Coronary artery angiography.}

\section{Competing interests}

The authors declare that they have no competing interest.

\section{Authors' contribution}

SYJ participated in the design of the study, collected and organized datas, analysed images, performed the statistical analysis and drafted the manuscript. YX collected, organized and analysed datas. ZY, GS and LH collected and organized datas. LT and XK analysed images. CL and WCW collected datas. QGX conceived of the study, and participated in its design and coordination and helped to draft the manuscript. All authors read and approved the final manuscript.

\section{Acknowledgements}

We acknowledge Professor Qi Guoxian who contributed towards the article by making substantial contributions to conception, design, drafting the manuscript and revising it critically for important intellectual content.

\section{Author details}

${ }^{1}$ Department of Cardiology of Aging, Department of Cardiology, The First Affiliated Hospital of China Medical University, NO.155 Nanjing North Street, Heping Ward, Shenyang 110001, China. '2Department of Radiology, The First Affiliated Hospital of China Medical University, Shenyang, China.

Received: 12 August 2013 Accepted: 31 July 2014

Published: 16 August 2014

\section{References}

1. Saugstad OD: Role of xanthine oxidase and its inhibitor in hypoxia: reoxygenation injury. Pediatrics 1996, 98:103-107.

2. Lee J, Sparrow D, Vokonas PS, Landsberg L, Weiss ST: Uric acid and coronary heart disease risk: evidence for a role of uric acid in the obesity-insulin resistance syndrome: the normative aging study. Am J Epidemiol 1995, 142:288-294.

3. Puig JG, Michán AD, Jiménez ML, Pérez De Ayala C, Mateos FA, Capitán CF, De Miguel E, Gijón JB: Female gout: clinical spectrum and uric acid metabolism. Arch Intern Med 1991, 151:726-732.

4. Puig JG, Ruilope LM: Uric acid as a cardiovascular risk factor in arterial hypertension. J Hypertens 1999, 17:869-872.

5. Chu NF, Wang DJ, Liou SH, Shieh SM: Relationship between hyperuricemia and other cardiovascular disease risk factors among adult males in Taiwan. Eur J Epidemiol 2000, 16:13-17.

6. Kim SY, Guevara JP, Kim KM, Choi HK, Heitjan DF, Albert DA: Hyperuricemia and coronary heart disease: a systematic review and meta-analysis. Arthritis Care Res (Hoboken) 2010, 62:170-180.

7. Yildiz A, Kaya Z: A crucial marker of cardiovascular diseases? Int J Cardiol 2012, 159:158.

8. Culleton BF, Larson MG, Kannel WB, Levy D: Serum uric acid and risk for cardiovascular disease and death: the Framingham heart study. Ann Intern Med 1999, 131:7-13.

9. Dutta A, Henley W, Pilling LC, Wallace RB, Melzer D: Uric acid measurement improves prediction of cardiovascular mortality in later life. J Am Geriatr Soc 2013, 61:319-326.

10. Niskanen LK, Laaksonen DE, Nyyssonen K, Alfthan G, Lakka HM, Lakka TA: Uric acid level as a risk factor for cardiovascular and all-cause mortality in middle-aged men: a prospective cohort study. Arch Intern Med 2004, 164:1546-1551.

11. Davies KJ, Sevanian A, Muakkassah-Kelly SF, Hochstein P: Uric acid-iron ion complexes. A new aspect of the antioxidant functions of uric acid. Biochem J 1986, 235:747-754

12. Hoieggen A, Alderman MH, Kjeldsen SE, Julius S, Devereux RB, De Faire U, Fyhrquist $F$, Ibsen $H$, Kristianson $K$, Lederballe-Pedersen $O$, Lindholm LH, Nieminen MS, Omvik P, Oparil S, Wedel H, Chen C, Dahlöf B, LIFE Study Group: LIFE study group. The impact of serum uric acid on cardiovascular outcomes in the LIFE study. Kidney Int 2004, 65:1041-1049.

13. Ndrepepa G, Braun S, King L, Fusaro M, Tada T, Cassese S, Hadamitzky M, Haase HU, Schömig A, Kastrati A: Uric acid and prognosis in angiography-proven coronary artery disease. Eur J Clin Invest 2013, 43:256-266

14. Sinan Deveci O, Kabakci G, Okutucu S, Tulumen E, Aksoy H, Baris Kaya E, Evranos B, Aytemir K, Tokgozoglu L, Oto A: The association between serum uric acid level and coronary artery disease. Int J Clin Pract 2010, 64:900-907.

15. Agatston AS, Janowitz WR, Hildner FJ, Zusmer NR, Viamonte M Jr, Detrano R: Quantification of coronary artery calcium using ultrafast computed tomography. J Am Coll Cardiol 1990, 15:827-832.

16. Austen WG, Edwards JE, Frye RL, Gensini GG, Gott VL, Griffith LS, McGoon DC, Murphy ML, Roe BB: A reporting system on patients evaluated for coronary artery disease. Report of the Ad Hoc committee for grading of coronary artery disease, council on cardiovascular surgery. American heart association. Circulation 1975, 51(suppl):5-40.

17. Cheng WY, Wolak A, Gutstein A, Gransar H, Wong ND, Dey D, Thomson LE, Hayes SW, Friedman JD, Slomka PJ, Berman DS: Low-density lipoprotein and noncalcified coronary plaque composition in patients with newly diagnosed coronary artery disease on computed tomographic angiography. Am J Cardiol 2010, 105:761-766.

18. Abada HT, Larchez C, Daoud B, Sigal-Cinqualbre A, Paul JF: MDCT of the coronary arteries: feasibility of low-dose CT with ECG-pulsed tube 
current modulation to reduce radiation dose. Am J Roentgen/ 2006, 186:S387-S390.

19. Mark DB, Berman DS, Budoff MJ, Carr JJ, Gerber TC, Hecht HS, Hlatky MA, Hodgson JM, Lauer MS, Miller JM, Morin RL, Mukherjee D, Poon M, Rubin GD, Schwartz RS: ACCF/ACR/AHA/NASCI/SAIP/SCAI/SCCT2010 expert consensus document on coronary computed tomographic angiography: a report of the American college of cardiology foundation task force on expert consensus documents. Circulation 2010, 121:2509-2543.

20. Kocaman SA, Sahinarslan A, Cemri M, Timurkaynak T, Boyaci B, Cengel A: Independent relationship of serum uric acid levels with leukocytes and coronary atheroscleroticburden. Nutr Metab Cardiovasc Dis 2009, 19:729-735.

21. loachimescu AG, Brennan DM, Hoar BM, Hazen SL, Hoogwerf BJ: Serum uric acid is an independent predictor of all-cause mortality in patients at high risk of cardiovascular disease: a preventive cardiology information system (PreCIS) database cohort study. Arthritis Rheum 2008, 58:623-630.

22. Moriarity JT, Folsom AR, Iribarren C, Nieto FJ, Rosamond WD: Serum acid uric and risk of coronary heart disease: atherosclerosis risk in communities (ARIC) study. Ann Epidemiol 2000, 10:136-143.

23. Strasak A, Ruttmann E, Brant L, Kelleher C, Klenk J, Concin H, Diem G, Pfeiffer K, Ulmer H, VHM\&PP Study Group: Serum uric acid and risk of cardiovascular mortality: a prospective long-term study of 83,683 Austrian men. Clin Chem 2008, 54:273-284.

24. Tavil Y, Kaya MG, Oktar SO, Sen N, Okyay K, Yazici HU, Cengel A: Uric acid level and its association with carotid intima-media thickness in patients with hypertension. Atherosclerosis 2008, 197:159-163.

25. Mutluay R, Deger SM, Bahadir E, Durmaz AO, Citil R, Sindel S: Uric acid is an important predictor for hypertensive early atherosclerosis. Adv Ther 2012, 29:276-286

26. Cipolli JA, Ferreira-Sae MC, Martins RP, Pio-Magalhães JA, Bellinazzi VR, Matos-Souza JR, Junior WN: Relationship between serum uric acid and internal carotid resistive index in hypertensive women: a cross-sectional study. BMC Cardiovasc Disord 2012, 12:52.

27. Liese AD, Hense HW, Löwel H, Döring A, Tietze M, Keil U: Association of serum uric acid with all-cause and cardiovascular disease mortality and inci-dent myocardial infarction in the MONICA Augsburg cohort world health organization monitoring trends and determinants in cardiovascular diseases. Epidemiology 1999, 10:391-397.

28. Fang J, Alderman MH: Serum uric acid and cardiovascular mortal-ity the NHANES I epidemiologic follow-up study, 1971-1992 national health and nutrition examination survey. JAMA 2000, 283:2404-2410.

29. Tuttle KR, Short RA, Johnson RJ: Sex differences in uric acid and risk factors for coronary artery disease. Am J Cardiol 2001, 87:1411-1414.

30. Detrano R, Guerci AD, Carr JJ, Bild DE, Burke G, Folsom AR, Liu K, Shea S, Szklo M, Bluemke DA, O'Leary DH, Tracy R, Watson K, Wong ND, Kronmal RA: Coronary calcium as a predictor of coronary events in four racial or ethnic groups. N Engl J Med 2008, 358:1336-1345.

31. Pletcher MJ, Tice JA, Pignone M: Using the coronary artery calcium score to predict coronary heart disease events: a systematic review and meta-analysis. Arch Internmed 2004, 164:1285-1292.

32. Rodrigues TC, Maahs DM, Johnson RJ, Jalal DI, Kinney GL, Rivard C, Rewers M, Snell-Bergeon JK: Serum uric acid predicts progression of subclinical coronary atherosclerosis in individuals without renal disease. Diabetes Care 2010, 33:2471-2473.

33. Mitsutake R, Miura S, Saku K: Association between coronary artery calcification score as assessed by multi-detector row computed tomography and upstroke time of pulse wave. Intern Med 2007, 46:1833-1836.

34. Coutinho Tde A, Turner ST, Peyser PA, Bielak LF, Sheedy PF, Kullo IJ: Associations of serum uric acid with markers of inflammation, metabolic syndrome, and subclinical coronary atherosclerosis. Am J Hypertens 2007, 20:83-89.

35. Atar Al, Yilmaz OC, Akin K, Selcoki Y, Er O, Eryonucu B: Association between gamma-glutamyltransferase and coronary artery calcification. Int J Cardiol 2012, Epub ahead of print.

36. Kaya EB, Yorgun H, Canpolat U, Hazırolan T, Sunman H, Ülgen A, Ates AH, Aytemir K, Tokgözoğlu L, Kabakcı G, Akata D, Oto A: Serum uric acid levels predict the severity and morphology of coronary atherosclerosis detected by multidetector computed tomography. Atherosclerosis 2010, 213:178-183.

37. Sano K, Kawasaki M, Ishihara Y, Okubo M, Tsuchiya K, Nishigaki K, Zhou X, Minatoguchi S, Fujita H, Fujiwara H: Assessment of vulnerable plaques causing acute coronary syndrome using integrated backscatter intravascular ultrasound. J Am Coll Cardiol 2006, 47:734-741.

38. Pundziute G, Schuijf JD, Jukema JW, Decramer I, Sarno G, Vanhoenacker PK, Boersma E, Reiber JH, Schalij MJ, Wijns W, Bax JJ: Evaluation of plaque characteristics in acute coronary syndrome: non-invasive assessment with multi-slice computed tomography and invasive evaluation with intravascular ultrasound radiofrequency data analysis. Eur Heart J 2008, 29:2373-2381

39. Gagliardi AC, Miname MH, Santos RD: Uric acid: a marker of increased cardiovascular risk. Atherosclerosis 2009, 202:11-17.

40. Yildiz A, Yilmaz R, Demirbag R, Gur M, Bas MM, Erel O: Association of serum uric acid level and coronary blood flow. Coron Artery Dis 2007, 18:607-613.

41. Kanellis J, Watanabe S, Li JH, Kang DH, Li P, Nakagawa T, Wamsley A, Sheikh-Hamad D, Lan HY, Feng L, Johnson RJ: Uric acid stimulates monocyte chemoattractant protein-1 production in vascular smooth muscle cells via mitogen-activated protein kinase and cyclooxygenase-2. Hypertension 2003, 41:1287-1293.

42. Kuwahata S, Hamasaki S, Ishida S, Kataoka T, Yoshikawa A, Orihara K, Ogawa M, Oketani N, Saihara K, Okui H, Shinsato T, Kubozono T, Ichiki H, Fujita S, Takumi T, Yoshino S, Nakazaki M, Miyata M, Tei C: Effect of uric acid on coronary microvascular endothelial function in women: association with eGFR and ADMA. J Atheroscler Thromb 2010, 17:259-269.

43. Athyros VG, Mikhailidis DP, Liberopoulos EN, Kakafika Al, Karagiannis A Papageorgiou AA, Tziomalos K, Ganotakis ES, Elisaf M: Effect of statin treatment on renal function and serum uric acid levels and their relation to vascular events in patients with coronary heart disease and metabolic syndrome: a subgroup analysis of the Greek atorvastatin and coronary heart disease evaluation (GREACE) study. Nephrol Dial Transplant 2007, 22:118-127.

doi:10.1186/1471-2261-14-101

Cite this article as: Sun et al:: A cross-sectional analysis of the relationship between uric acid and coronary atherosclerosis in patients with suspected coronary artery disease in China. BMC Cardiovascular Disorders 2014 14:101.

\section{Submit your next manuscript to BioMed Central and take full advantage of:}

- Convenient online submission

- Thorough peer review

- No space constraints or color figure charges

- Immediate publication on acceptance

- Inclusion in PubMed, CAS, Scopus and Google Scholar

- Research which is freely available for redistribution 